\title{
Visualization of large vessel occlusion, clot extent, and collateral supply using volume perfusion flat detector computed tomography in acute stroke patients
}

Acta Radiologica

2019, Vol. 60(II) I504-I5II

(C) The Foundation Acta Radiologica 2019

Article reuse guidelines: sagepub.com/journals-permissions DOI: $10.1177 / 0284185$ II 9836220 journals.sagepub.com/home/acr (S)AGE

\author{
Philip Hoelter' (D, Philipp Goelitz', Stefan Lang', \\ Hannes Luecking', Bernd Kalmuenzer², Tobias Struffert' and \\ Arnd Doerfler'
}

\begin{abstract}
Background: Flat detector computed tomography angiography (FDCTA) can be reconstructed from volume perfusion flat detector computed tomography (VP-FDCT). Thus, CTA equivalent stroke imaging might be feasible within the angio suite.

Purpose: To evaluate the diagnostic accuracy of FDCTA in detecting large vessel occlusion (LVO) and collateral supply in acute stroke patients.

Material and Methods: Sixteen patients with LVO of the anterior circulation were analyzed retrospectively. Each patient underwent a multimodal CT stroke protocol, subsequent VP-FDCT, and digital subtraction angiography (DSA) for endovascular stroke therapy. Angiographic images reconstructed secondarily from VP-FDCT were evaluated with regard to visualization of LVO, Collateral Score (CS), Clot Burden Score (CBS), and image quality.

Results: Image quality of FDCTA was sufficient for diagnosis with a strong correlation between CTA and FDCTA (median score: CTA $=4 \pm 0.447 ; \mathrm{FDCTA}=4 \pm 0.5$ ). Detection of LVO could be achieved with high sensitivity and specificity for FDCTA and CTA $(97.9 \%, 95 \%$ confidence interval $[\mathrm{Cl}]=96.0-99.9 ; 92.6 \%, 95 \% \mathrm{Cl}=84.3-100.0$ vs. $96.8 \%, 95 \% \mathrm{Cl}=93.2-100.0 ; 86.3 \%, 95 \% \mathrm{Cl}=88.2-95.2)$. CBS and $\mathrm{CS}$ assessment showed no significant difference between FDCTA and CTA for both readers (reader $\mathrm{I}_{\mathrm{CBS}}: P=0.75 \mathrm{I}$, reader $\mathrm{I}_{\mathrm{CS}}: P=0.7 \mathrm{I}$; reader $2_{\mathrm{CBS}}: P=0.164$; reader2 $\left.2_{C S}: P=0.582\right)$ and an excellent interrater agreement $\left(C T A_{C B S} I C C=0.984, \quad F D C T A_{C B S} I C C=0.95 I\right.$; $C T A_{C S} I C C=0.754, F D C T A_{C S} I C C=0.789$ ).

Conclusion: FDCTA, reconstructed from VP-FDCT data, allows reliable detection of ICA or MCA MI segment occlusion comparable to CTA and may provide information concerning the clot extent with sufficient image quality.
\end{abstract}

\section{Keywords}

Central nervous system, interventional, stroke, flat detector computed tomography

Date received: 2 August 2018; accepted: 10 February 2019

\section{Introduction}

In neuroangiographic imaging, flat detector computed tomography (FDCT) plays an increasing role as it allows the acquisition of computed tomography (CT) such as images within the angio suite $(1,2)$. By a rotational acquisition from an angular range of approximately $200^{\circ}$ a three-dimensional (3D) volume can be derived, which can be post-processed and evaluated like multislice CT datasets (3). Thereby, cross-sectional
'Department of Neuroradiology, University Hospital Erlangen, Erlangen, Germany

${ }^{2}$ Department of Neurology, University Hospital Erlangen,

Erlangen, Germany

\section{Corresponding author:}

Philip Hoelter, Department of Neuroradiology, University Hospital Erlangen, Schwabachanlage 6, 91054 Erlangen, Germany.

Email: philip.hoelter@uk-erlangen.de 
imaging of the brain can be performed within the angio suite and intracerebral hemorrhage (ICH) or subarachnoid hemorrhage (SAH) can be sufficiently recognized even though image quality is inferior to CT $(2,4)$.

Moreover, it has been shown that using volume perfusion FDCT (VP-FDCT), parenchymal blood volume (PBV) maps can be generated, which provide an approximation of the cerebral blood volume (CBV), a key parameter of brain perfusion $(5,6)$. In the treatment of patients with acute ischemic stroke (AIS), workflow analysis of the ESCAPE trial showed that imaging-toreperfusion time is an important predictor of outcome (7). The possibility to perform equivalent diagnostic imaging within the angio suite could shorten imagingto-reperfusion time and speed up the workflow $(8,9)$. Robust imaging of large vessel occlusion (LVO) and collateral supply out of VP-FDCT datasets, which are comparable to computed tomography angiography (CTA), would help to implement a "one-stop" approach in the work-up of ischemic stroke within the angio suite. However, reliable imaging of the intracranial vasculature, the clot burden, and the collateral supply in AIS is warranted (10-17).

We aimed to compare the diagnostic accuracy of flat detector computed tomography angiography (FDCTA), reconstructed from VP-FDCT, with CTA.

\section{Material and Methods}

\section{Patients}

Informed consent was gathered by the patients or legal representatives according to local law and regulations. Institutional review board approval was obtained before the commencement of this study and this study was performed in accordance with the ethical standards laid down in the 1964 Declaration of Helsinki and its later amendments.

A total of 16 patients were included retrospectively. Inclusion criteria were NIHSS $>3$, symptom onset within the last $6 \mathrm{~h}$ and a suspected occlusion of the anterior circulation. In all patients, occlusion of the internal carotid artery (ICA) or the middle cerebral artery (MCA) was suspected. All patients underwent multimodal CT, VP-FDCT, and endovascular treatment (EVT). Baseline characteristics are listed in Table 1.

\section{Multimodal CT}

CT was performed using a 128-section scanner (Somatom Definition AS+; Siemens Healthineers, Forchheim, Germany). A multimodal stroke protocol was used in the following order: non-enhanced
Table I. Baseline characteristics.

\begin{tabular}{ll}
\hline Demographic and clinical characteristics & \\
Gender* (female) & $8(50 \%)$ \\
Age $^{\dagger}$ (years) & $70 \pm 13$ \\
Pre-mRS* $^{*}$ & $0(0-1)$ \\
Hypertension* & $15(93.8 \%)$ \\
Diabetes* & $4(25 \%)$ \\
Hypercholesterolemia* & $6(37.5 \%)$ \\
Atrial fibrillation* & $1 \mathrm{I}(68.8 \%)$ \\
Admission status & \\
NIHSS & $15(13-17)$ \\
Onset-to-image time (min) & $\ddagger$ \\
I.v. lysis treatment* & $99.5(79-23 \mathrm{I})$ \\
General anesthesia before EVT* & $13(8 \mathrm{I} .3 \%)$ \\
\end{tabular}

*Values are presented as $\mathrm{n}(\%)$.

tValues are presented as mean \pm SD.

$\ddagger$ Values are presented as median (interquartile range).

mRS, modified Rankin Scale; NIHSS, National Institutes of Health Stroke Scale; I.v., intravenous; EVT, endovascular treatment.

computed tomography (NECT); computed tomography perfusion (CTP); and CTA.

CTA was performed in a caudocranial direction. Coverage in the $\mathrm{z}$-axis was from the aortic arch to the cranial vertex $(120 \mathrm{kV}, 160 \mathrm{mAs}$, collimation $=$ $128 \times 0.6 \mathrm{~mm}$, rotation time $=0.3 \mathrm{~s})$. A total of $60 \mathrm{~mL}$ contrast agent (Imeron ${ }^{\circledR}$ 400, Bracco Imaging, Konstanz, Germany) was injected via an 18-gauge cubital-vein-cannula at a rate of $5 \mathrm{~mL} / \mathrm{s}$, followed by $50 \mathrm{~mL}$ of saline flush. Bolus tracking was performed in the ascending aorta and image acquisition started $4 \mathrm{~s}$ after contrast exceeding $100 \mathrm{HU}$ (18).

CTP was performed in caudocranial direction $(80 \mathrm{kV}, 80 \mathrm{mAs}$; z-axis coverage $=96 \mathrm{~mm} ; 1$ scan every $1.5 \mathrm{~s}$; 67.98 -s scan period; 2 -s delay after contrast injection). A total of $30 \mathrm{~mL}$ contrast agent $\left(\right.$ Imeron $^{\circledR}$ 400, Bracco Imaging, Konstanz, Germany) was injected via an 18-gauge cubital-vein cannula at a rate of $5 \mathrm{~mL} / \mathrm{s}$, followed by $50 \mathrm{~mL}$ of saline flush.

\section{FDCT/Digital subtraction angiography}

Subsequent FDCT was performed at the same angiography systems (Axiom Artis dBA or Axiom Artis Zee Zeego; Siemens Healthineers, Forchheim, Germany) in one session with digital subtraction angiography (DSA).

VP-FDCT (Neuro-PBV IR; Siemens Healthcare, Forchheim, Germany) was performed immediately before EVT. Each acquisition consisted of a mask run, followed by a fill run. Rotation time per run was $8 \mathrm{~s}(70 \mathrm{kV}, 616 \times 480$ matrix, projection on $30 \times 40 \mathrm{~cm}$ flat panel size, $200^{\circ}$ total angle, $0.5^{\circ} /$ frame, 400 frames total, dose per frame $0.36 \mu \mathrm{Gy}$ ). Image acquisition was started by using the "bolus watching" method (5). 
A total of $60 \mathrm{~mL}$ contrast agent (Imeron ${ }^{\circledR} 400$, Bracco Imaging, Konstanz, Germany) was injected via an 18-gauge cubital-vein cannula at a rate of $5 \mathrm{~mL} / \mathrm{s}$, followed by $50 \mathrm{~mL}$ of saline flush. For patients' head fixation, a carbon head holder with soft tapes was used.

\section{Post-processing of VP-FDCT and CTA}

Post-processing of VP-FDCT images was performed using a dedicated workstation (syngo XWP, Siemens Healthineers, Forchheim, Germany). The mode "native fill," kernel type "HU," and image impression "normal" were used (19).

Angiographic maximum intensity projection (MIP) reconstructions of each VP-FDCT dataset and CTA dataset were generated in transverse and coronal orientation (slice thickness $=1 \mathrm{~mm}$; spacing $=1 \mathrm{~mm}$ ).

\section{Image analysis}

All FDCTA and CTA datasets were evaluated separately and in random order by two independent neuroradiologists (two and five years of experience) blinded to clinical data. Analysis of DSA as reference was performed afterwards in consensus by the same two neuroradiologists.

Visual (qualitative) analysis of both FDCTA and CTA MIP reconstructions was performed in consensus using a 4-point scale, in the style previously described by Hinkmann et al. (20): $1=$ poor quality, vasculature not definable, no information; 2 = fair quality, vasculature suboptimal definable, clear loss of information; $3=$ good quality, vasculature can be defined without substantial loss of information; and $4=$ excellent quality, exact definable vasculature, no loss of information.

The following vascular segments were screened for LVO: distal ICA left/right, segments $\mathrm{C} 6 / \mathrm{C} 7$, respectively, according to the Bouthillier classification; proximal M1 left/right; distal M1 left/right; M2-M3 left/right. Vessel occlusion was described using a 2-point scale for FDCTA, CTA, and DSA: $0=$ vessel occluded; and $1=$ vessel open.

Clot Burden Score (CBS) in FDCTA and CTA datasets was evaluated, as described by Tan et al. (17). Briefly, the CBS describes the extent of thromboembolic occlusion of seven vessel segments of the anterior circulation: the infra- and supraclinoid ICA, the proximal and distal M1 segment, two M2 segments, and the A1 segment of the anterior cerebral artery (ACA). The score applies a scale of $0-10$, implying a clot absence by a score of 10 or a multivessel occlusion by a score of 0 . If there is a thrombus in the supraclinoid ICA, the proximal M1 segment, or the distal M1 segment, for each a score of 2 is subtracted by 10 . If there is a thrombus in the infraclinoid ICA, the ACA, or in the superior or inferior M2 segment, for each a score of 1 is subtracted by 10 . It is irrelevant if the thrombus is completely or partially occlusive. The CBS was evaluated using MIP reconstructions of the FDCTA and CTA datasets. None of our participants presented an occlusion of the ACA or the infraclinoid ICA.

Collateral Score (CS) was surveyed using FDCTA and CTA datasets as described by Tan et al. (17): $0=$ no collateral supply of the occluded MCA territory; $1=$ collateral supply $>0 \%$ but $\leq 50 \%$ of the occluded MCA territory; $2=$ collateral supply $>50 \%$ but $\leq 100 \%$ of the occluded MCA territory; and $3=100 \%$ collateral supply of the occluded MCA territory.

\section{Statistical analysis}

For statistical analysis, Spearman correlation was used to correlate LVO between FDCTA, CTA, and DSA. Segment-based sensitivity and specificity of LVO detection was calculated. To compare CBS and CS, a paired t-test was used. A $P$ value $\leq 0.05$ was considered as significant.

To assess the interrater agreement of LVO, CBS, and $\mathrm{CS}$, the interrater correlation coefficient (ICC) was used. ICC estimates and their 95\% confident intervals (CI) were based on a single rater, absolute-agreement, twoway mixed-effects model (21). Interpretation of average ICC values was as follows: poor $\leq 0.40$; fair $=0.40-0.59$; good $=0.60-0.74$; and excellent $=0.75-1.00$.

All statistical analyses were performed using commercially available software (IBM ${ }^{\circledR}$ SPSS $^{\circledR}$ Statistics Version 19, Chicago, IL, USA).

\section{Results}

\section{Qualitative analysis}

Overall quality was sufficient to define the vasculature confidently (Table 2). Intracranial vessel anatomy could be identified exactly for $12 / 16(75 \%)$ CTA datasets $(4$, range $=3.25-4)$ and for $10 / 16(62.5 \%)$ FDCTA

Table 2. Qualitative scoring of FDCTA and CTA.

\begin{tabular}{lllll}
\hline & \multicolumn{4}{l}{ Grades of quality } \\
\cline { 2 - 5 } & $\mathrm{I}$ & 2 & 3 & 4 \\
\hline $\begin{array}{llll}\text { Modality } \\
\text { CTA }\end{array}$ & $0(0)$ & $0(0)$ & $4(25)$ & $12(75)$ \\
FDCTA & $0(0)$ & $0(0)$ & $6(37.5)$ & $10(62.5)$ \\
\hline
\end{tabular}

Values are presented as $\mathrm{n}(\%)$

Analysis was performed by two neuroradiologists in consensus. I = poor quality, vasculature not definable, no information; 2 = fair quality, vasculature suboptimal definable, clear loss of information; $3=$ good quality, vasculature can be defined without substantial loss of information; $4=$ excellent quality, exact definable vasculature, no loss of information. 
datasets (4, range $=3-4)$. No dataset showed suboptimal or missing vessel delineation.

Correlation between CTA and FDCTA was strong $(\mathrm{r}=0.745 ; P=0.001)$.

\section{Large vessel occlusion}

A total of 384 vessel segments were scored (128 by FDCTA, 128 by CTA, 128 by DSA). LVO could be detected reliably in all patients.

Analysis of the DSA as reference revealed ICA occlusion in 4/16 patients ( $2 / 4$ left-sided, $2 / 4$ rightsided). Of these 16 patients, 12 presented with an MCA occlusion. Therefore, the M1 segment was primarily occluded in $10 / 12$ patients (3/10 left-sided, 7/10 right-sided), whereas there was a primarily occlusion of the M2 segment in 2/12 patients (1/2 left-sided, $1 / 2$ right-sided). Overall, 41/128 vessel segment occlusions were scored in the DSA.

Both readers detected occlusions in 38/128 (29.7\%) vessel segments when examining CTA datasets.

Reader 1 examined 42/128 (32.8\%) vessel segment occlusions when scoring FDCTA datasets, whereas reader 2 scored 34/128 (26.6\%) occlusions.

Overall correlation in detecting LVO between CTA and FDCTA was excellent for reader 1 (mean $r=0.894$ \pm 0.07 ) and reader 2 (mean $r=0.767 \pm 0.249$ ). However, there was no correlation in the right $\mathrm{M} 2 / 3$ segment by reader $2(\mathrm{r}=0.289, P=0.278)$.

The correlation between FDCTA and DSA was also excellent for reader 1 (mean $\mathrm{r}=0.867 \pm 0.094$ ) and reader 2 (mean $r=0.839 \pm 0.447$ ), with no correlation in detecting vessel occlusion in the right M2/3 segment by reader $2(\mathrm{r}=0.447, P=0.082)$. For both readers, the mean sensitivity in detecting LVO using CTA was $96.8 \%(95 \% \mathrm{CI}=93.2-100.0)$, the mean specificity was $86.3 \%(95 \% \mathrm{CI}=88.2-95.2)$, the positive predictive value (PPV) was $92.8 \%(95 \% \mathrm{CI}=88.2-97.3)$, and the negative predictive value (NPV) was $95.7 \%(95 \%$ $\mathrm{CI}=90.5-100.0$ ). For FDCTA, the mean sensitivity was $97.9 \%(95 \% \mathrm{CI}=96.0-99.9)$, the specificity was $92.6 \%(95 \% \mathrm{CI}=84.3-100.0)$, the PPV was $94.4 \%$ $(95 \% \mathrm{CI}=87.3-100.0)$, and the NPV was $93.8 \%$ $(95 \% \mathrm{CI}=87.4-100.0)$.

Interrater agreement was excellent for both CTA evaluation (mean $\mathrm{ICC}=0.93 \pm 0.092$ ) and FDCTA assessment (mean ICC $=0.932 \pm 0.111$ ).

\section{Clot Burden Score}

The median CBS was $6($ mean $=5.0 \pm 2.422$, range $=1-8)$ in CTA measured by reader 1. In FDCTA datasets, the median CBS was 6 (mean $=5.06 \pm 2.62$, range $=1-9)$. Reader 2 evaluated a median CBS of 6 in CTA $($ mean $=4.75 \pm 2.35, \quad$ range $=1-8) \quad$ and FDCTA (mean $=5.2 \pm 2.43$, range $=1-8)$. There was no significant difference between CTA and FDCTA for both readers (reader 1: $P=0.751$; reader 2: $P=0.164$ ).

Interrater agreement was excellent for both CTA evaluation $(\mathrm{ICC}=0.984)$ and FDCTA assessment $(\mathrm{ICC}=0.951)$.

\section{Collateral Score}

Median CS was $1($ mean $=1.56 \pm 0.727$, range $=1-3)$ as assessed in CTA and FDCTA datasets (mean $=1.63$ \pm 0.619 , range $=1-3$ ) by reader 1 . Reader 2 scored a median CS of 1 in CTA (mean $=1.25 \pm 0.683$, range $=0-3$ ) and a median of 1 (mean $=1.4 \pm 0.986$, range $=0-3$ ) for the FDCTA datasets.

There was no significant difference between CTA and FDCTA for both readers (reader 1: $P=0.718$; reader 2: $P=0.582$ ).

Interrater agreement was excellent in CTA assessment $\quad(\mathrm{ICC}=0.754)$ and FDCTA assessment $(\mathrm{ICC}=0.789)$.

\section{Discussion}

In the present study, we demonstrated that detection of LVO and evaluation of collateral status and clot burden is reliably possible when using VP-FDCT. Secondarily reconstructed FDCTA offers an image quality for confident diagnosis of LVO in patients with AIS (Figs. 1 and 2).

Available data revealed that image quality of FDCT is sufficient to exclude ICH (2). In addition, VP-FDCT is a technique providing information on perfusion status highly sensitive in detecting perfusion deficits (6). To our knowledge our study is the first specifically designed to assess image quality and diagnostic accuracy of FDCTA, generated out of VP-FDCT data, compared to CTA in patients with AIS due to LVO.

In our qualitative assessment, FDCTA was sufficient to delineate intracerebral vessel anatomy comparable to CTA. Saake et al. showed that the depiction of cerebral vasculature using FDCTA was significantly superior compared to CTA, a result we could not observe in our trial (22). However, in their study they used a different FDCT program (10s DRH) which is dedicated to image intracranial vasculature. In addition, the performed scan was triggered to the arterial filling. The program we used (Neuro-PBV IR) is primarily designed to measure the $\mathrm{CBV}$, triggered to the "steady state." Moreover, their sample size was even smaller than ours.

When compared to CT, FDCT is clearly inferior with regards to the assessment of brain parenchyma or signs of early infarction, Alberta Stroke Program Early CT Score (ASPECTS), respectively (23). 


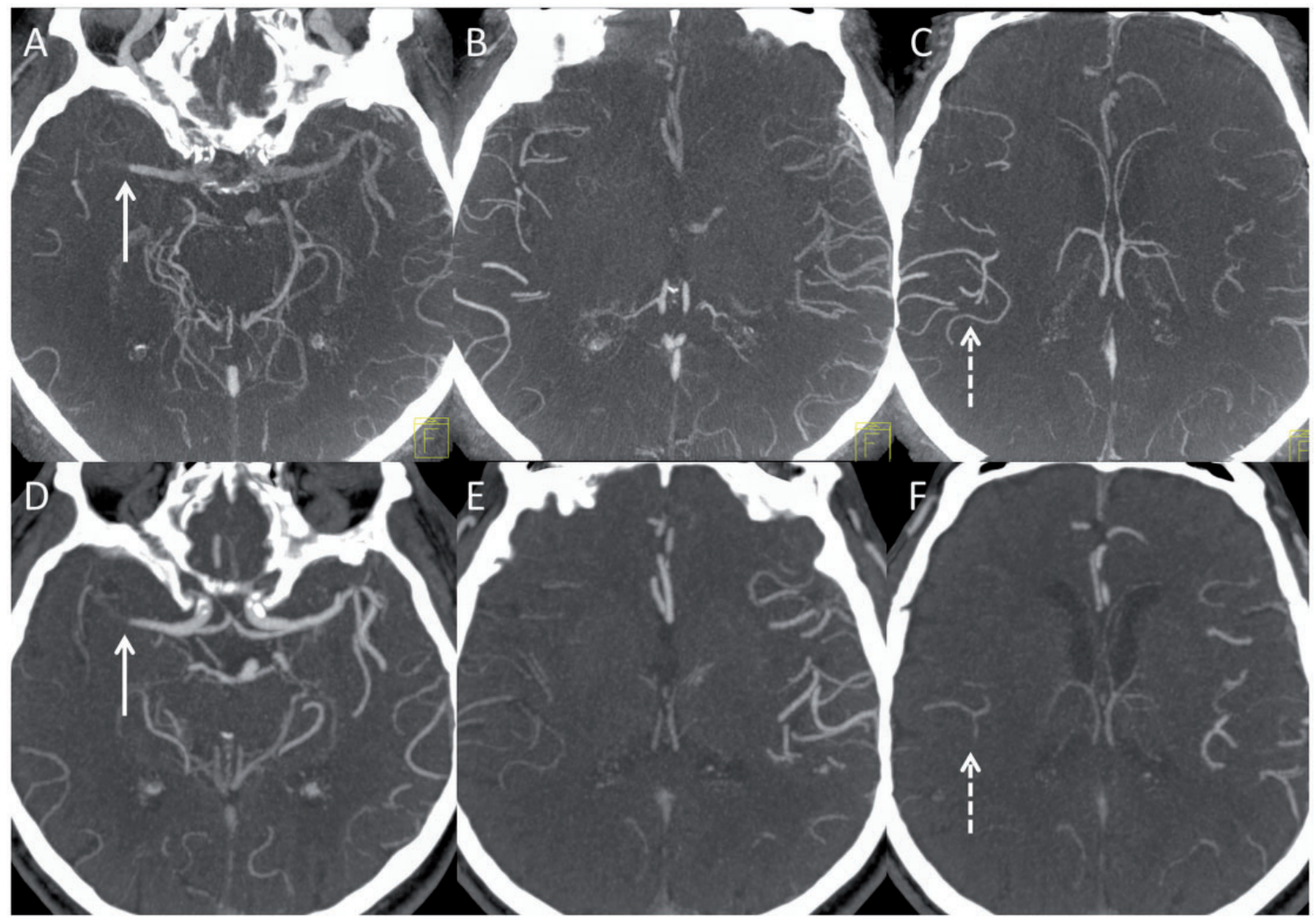

Fig. I. Angiographic MIPs in the axial plain of an 85-year-old male patient before undergoing thrombectomy (2.5-h time-window, NIHSS of 18 at admission). Images from the same planes were reconstructed from VP-FDCT datasets (a-c) and from CTA datasets $(d-f)$. ASPECT score as evaluated on CT images was 9 . Both readers scored 6 for the CBS in both modalities and a CS of I for CT and 2 for FDCT data. Continuous arrows: occlusion of the distal MI segment of the MCA. Dashed arrows: filling of the MCA territory via collaterals. Due to the latter phase of contrast, more filling can be delineated witch VP-FDCT (c) when compared to CTA (f).

Although it is capable of SAH depiction, the quality of FDCT images is inferior when compared to CT (4). Delineation of small infratentorial SAH is particularly more challenging (9).

However, in line with recent literature presenting FDCTA as a feasible method in imaging intracerebral vasculature, our results showed an excellent correlation between FDCTA and CTA in detecting LVO (23). For the right $\mathrm{M} 2 / 3$ segment, there was no correlation between FDCTA, CTA, and DSA, respectively, when analyzed by reader 2 . This might be due to motion artefacts despite a carefully head fixation during the rotational acquisition, as FDCT is sensitive to head motion. On the other hand, a minor experience of the reader might have contributed to this result as well, revealing that training is demanded for this highly specialized method.

Efforts to establish the neuroimaging triage in patients with AIS within the angio suite demand for a sensitive and specific LVO detection. To our knowledge, this is the first study describing similar sensitivity and specificity of FDCTA and CTA in detecting LVO in AIS, in line with current literature (24). In our trial, FDCTA showed a high level of sensitivity and specificity for the detection of LVO and we could verify the accuracy of FDCTA in ICA or M1 occlusions. However, there are important differences on how FDCTA and CTA are acquired. In CTA, bolus tracking was applied, whereas in VP-FDCT, the bolus watching method was used. For the CBV it is important that there is contrast in the whole brain parenchyma, the so-called "steady state.". This is why the delay between injection and start of the second rotation is in the range of 16-19 s, depending on the opacification of the dural sinus. The acquisition of the CTA images is much quicker, as it is triggered by the opacification of the ascending aorta. In patients with LVO, brain areas depending on collateral supply might show no or only fair contrast enhancement when imaging of the vasculature is performed too early. Although being a static acquisition, VP-FDCT shows a later phase of contrast compared to single-phase CTA. Together with the 


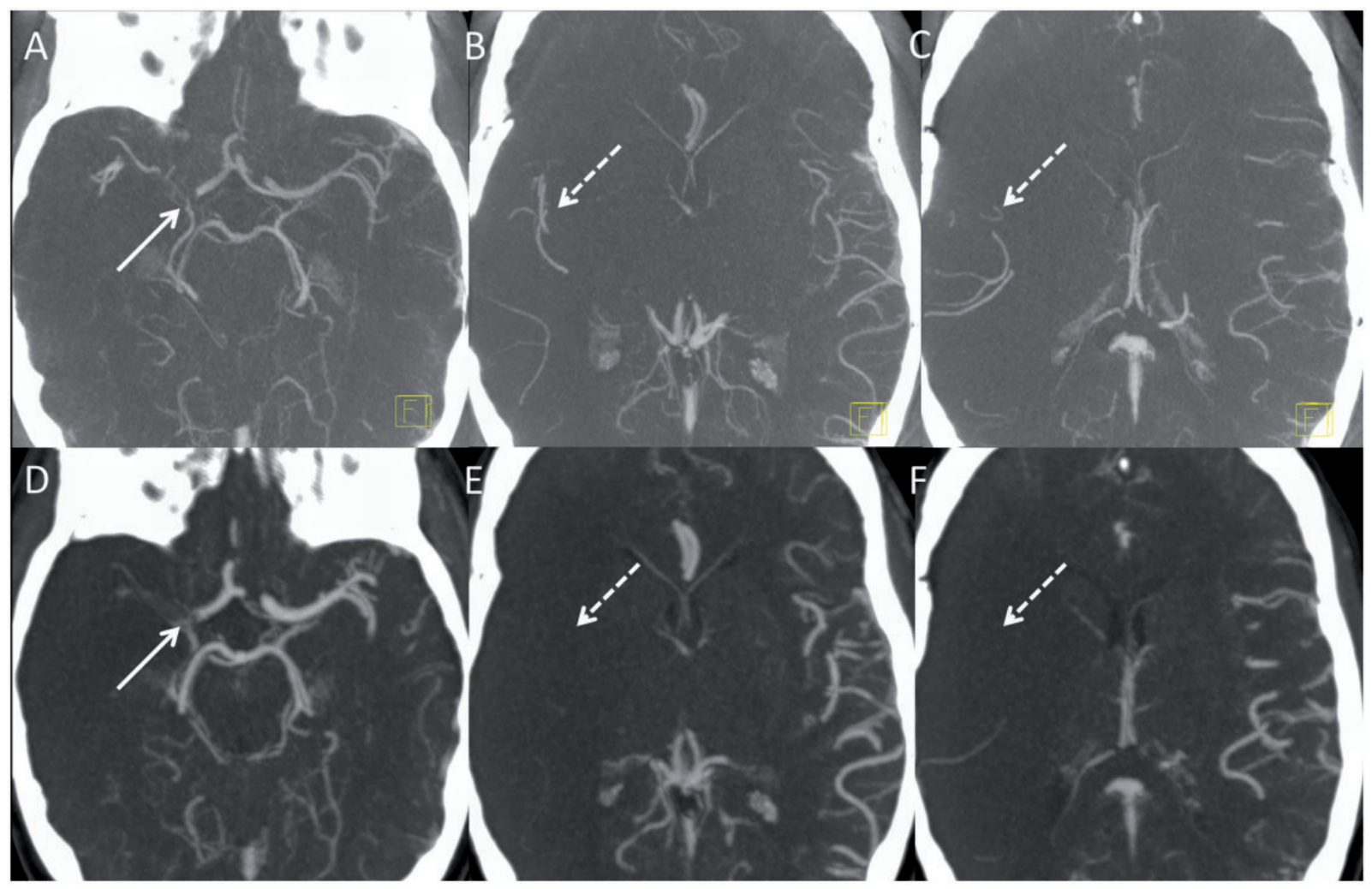

Fig. 2. Angiographic MIPs in axial plain of a 74-year-old female patient before undergoing thrombectomy (4-h time-window, NIHSS of 14 at admission). Images from the same planes were reconstructed from VP-FDCT datasets $(a-c)$ and from CTA datasets ( $d-f$ ). ASPECT score as evaluated on CT images was 4. A CBS of 6 was assessed in both modalities by both readers. A CS of I was assessed by both readers for CT and FDCT data. Continuous arrows: occlusion of the carotid-T of the distal ICA. Dashed arrows: in the VPFDCT datasets, augmented filling of the MCA territory via collaterals can be defined $(b, c)$ when compared to the CTA data (e, f).

rather small patient collective, this might explain the lower sensitivity and specificity values for CTA when compared to FDCTA.

VP-FDCT offers whole brain coverage, which enables the validation of CBS and CS. In contrast to the CBS, the correlation of the CS with CTA data was moderate. As explained before, the later phase of contrast compared to single-phase CTA might explain the different and non-correlating CS and might have misled to an overall slightly higher CS rating in FDCTA (Figs. 1 and 2). As VP-FDCT took place after CTA, this might have an impact on imaging the collateral status. However, we could not observe residual vessel contrast within the mask run datasets. Therefore, we think that this effect is rather negligible in comparison to the different principal of contrast application as described earlier. The later phase of contrast in intracranial vessels when using VP-FDCT might rather be an advantage over single phase CTA. It has already been shown that dynamic imaging, fourdimensional (4D)-CTA with an even higher resolution in time than mCTA, is superior to monophasic CTA in defining CBS and CS, as it offers a more accurate vessel distinction $(18,25)$. Multiphase CTA (mCTA) is a technique already known for better collateral evaluation compared to single-phase CTA $(26,27)$. Thus, FDCTA might correlate better with mCTA. New, yet prototype-based, 4D-FDCTA could probably improve the delineation of collateral supply and the description of the clot (28). Yang et al. recently reported a comprehensive evaluation of vessel occlusions by using time-resolved FDCTA (29). However, these results are based on not commercially available prototypes, where issues such as $\mathrm{C}$-arm rotational speed, patient safety, and radiation exposure first need to be addressed. This trial, however, is based on data acquired with a product already available and approved. Post-processing is implemented in clinical routine and reconstruction time is comparable to CTA (approximately 3-5 $\mathrm{min}$ ) but depends on the operators' experience.

Radiation exposure might be comparable with an effective dose of $2.3 \mathrm{mSv}$ for VP-FDCT and 3.6-5 $\mathrm{mSv}$ for CTP (30). Due to the rotation angle of $200^{\circ}$, dosimetry of FDCT is not equivalent to CT though and can rather be considered as approximation. 
Moreover, in this FDCT approach, information on dynamic perfusion parameters, as offered by CTP, is missing. Static, already available VP-FDCT might not replace multimodal CT. However, as AIS is a dynamic event, especially in outpatients when prolonged imaging-to-groin-puncture time is conceivable, VP-FDCT might be used as an instrument to detect both changes in $\mathrm{CBV}$ that describe the infarct core and clot extent (5).

The availability of cross-sectional angiographic images at the site of treatment emphasizes that AIS diagnostic work-up and subsequent EVT is possible within the same room. Time is a crucial parameter and a primary determinant of outcome in patients with AIS due to LVO and thus a fast workflow is warranted (7). FDCT could offer the possibility to perform the whole neuroimaging triage within the angio suite. Patients with suspected LVO would be transferred directly to the angio suite, where hemorrhage would be excluded before invasive DSA. LVO, brain vasculature, and infarct core were visualized immediately before EVT would take place. As a result, imagingto-groin-puncture time and imaging-to-reperfusion time could be shortened substantially.

Our study has several limitations. First, it is retrospectively designed, which bears the risk of selection bias. Second, possible effects of movement artefacts were not investigated. As patients' heads were fixed, no relevant artefacts were detected. However, our sample size is relatively small and the majority of our patients were under general anesthesia. As FDCT is sensitive to motion artefacts, patients under conscious sedation could show more movement of the head, resulting in an impaired image quality. Third, our study lacks an evaluation of patients with AIS due to LVO of the posterior circulation. As the posterior cranial fossa is a challenging region for imaging a dedicated evaluation is warranted.

In conclusion, our results suggest that FDCTA reconstructed from VP-FDCT allows reliable detection of ICA or MCA M1 segment occlusion and a diagnostic accuracy comparable to CTA. In addition, it may provide information concerning the clot extent and collateral supply.

\section{Declaration of conflicting interests}

The author(s) declared no potential conflicts of interest with respect to the research, authorship, and/or publication of this article.

\section{Funding}

The author(s) received no financial support for the research, authorship, and/or publication of this article.

\section{ORCID iD}

Philip Hoelter (D) http://orcid.org/0000-0001-9768-9630

\section{References}

1. Soderman M, Babic D, Holmin S, et al. Brain imaging with a flat detector $\mathrm{C}$-arm: Technique and clinical interest of XperCT. Neuroradiology 2008;50:863-868.

2. Struffert T, Eyupoglu IY, Huttner HB, et al. Clinical evaluation of flat-panel detector compared with multislice computed tomography in 65 patients with acute intracranial hemorrhage: initial results. Clinical article. J Neurosurg 2010;113:901-907.

3. Kamran M, Nagaraja S, Byrne JV. C-arm flat detector computed tomography: the technique and its applications in interventional neuro-radiology. Neuroradiology 2010;52:319-327.

4. Doelken M, Struffert T, Richter G, et al. Flatpanel detector volumetric CT for visualization of subarachnoid hemorrhage and ventricles: preliminary results compared to conventional CT. Neuroradiology 2008;50:517-523.

5. Struffert T, Deuerling-Zheng Y, Kloska S, et al. Cerebral blood volume imaging by flat detector computed tomography in comparison to conventional multislice perfusion CT. Eur Radiol 2011;21:882-889.

6. Fiorella D, Turk A, Chaudry I, et al. A prospective, multicenter pilot study investigating the utility of flat detector derived parenchymal blood volume maps to estimate cerebral blood volume in stroke patients. J Neurointerv Surg 2014;6:451-456.

7. Menon BK, Sajobi TT, Zhang Y, et al. Analysis of workflow and time to treatment on thrombectomy outcome in the Endovascular Treatment for Small Core and Proximal Occlusion Ischemic Stroke (ESCAPE) randomized, controlled trial. Circulation 2016;133:2279-2286.

8. Struffert T, Deuerling-Zheng Y, Kloska S, et al. Dynamic angiography and perfusion imaging using flat detector CT in the angiography suite: a pilot study in patients with acute middle cerebral artery occlusions. Am J Neuroradiol 2015;36:1964-1970.

9. Doerfler A, Golitz P, Engelhorn T, et al. Flat-panel computed tomography (DYNA-CT) in neuroradiology. From high-resolution imaging of implants to one-stopshopping for acute stroke. Clin Neuroradiol 2015;25 (Suppl. 2):291-297.

10. Fanou EM, Knight J, Aviv RI, et al. Effect of collaterals on clinical presentation, baseline imaging, complications, and outcome in acute stroke. Am J Neuroradiol 2015;36:2285-2291.

11. Gersing AS, Schwaiger BJ, Kleine JF, et al. Clinical outcome predicted by collaterals depends on technical success of mechanical thrombectomy in middle cerebral artery occlusion. J Stroke Cerebrovasc Dis 2017;26:801-808.

12. Boers AMM, Sales Barros R, Jansen IGH, et al. Value of quantitative collateral scoring on $\mathrm{CT}$ angiography in patients with acute ischemic stroke. Am J Neuroradiol 2018;39:1074-1082. 
13. Treurniet KM, Yoo AJ, Berkhemer OA, et al. Clot Burden Score on baseline computerized tomographic angiography and intra-arterial treatment effect in acute ischemic stroke. Stroke 2016;47:2972-2978.

14. Singer OC, Berkefeld J, Nolte $\mathrm{CH}$, et al. Collateral vessels in proximal middle cerebral artery occlusion: the ENDOSTROKE study. Radiology 2015;274:851-858.

15. Riedel CH, Zimmermann P, Jensen-Kondering U, et al. The importance of size: successful recanalization by intravenous thrombolysis in acute anterior stroke depends on thrombus length. Stroke 2011;42:1775-1777.

16. Grech R, Galvin PL, Power S, et al. Outcome prediction in acute stroke patients considered for endovascular treatment: a novel tool. Interv Neuroradiol 2014;20:312-324.

17. Tan IY, Demchuk AM, Hopyan J, et al. CT angiography clot burden score and collateral score: correlation with clinical and radiologic outcomes in acute middle cerebral artery infarct. Am J Neuroradiol 2009;30:525-531.

18. Kaschka IN, Kloska SP, Struffert T, et al. Clot Burden and collaterals in anterior circulation stroke: differences between single-phase CTA and multi-phase 4D-CTA. Clin Neuroradiol 2016;26:309-315.

19. Ott S, Struffert T, Saake M, et al. Influence of different reconstruction parameters in the visualization of intracranial stents using $\mathrm{C}$-arm flat panel CT angiography: experience in an animal model. Acta Radiol 2016;57:233-240.

20. Hinkmann FM, Voit HL, Anders K, et al. Ultra-fast carotid CT-angiography: low versus standard volume contrast material protocol for a 128-slice CT-system. Invest Radiol 2009;44:257-264.

21. Koo TK, Li MY. A guideline of selecting and reporting intraclass correlation coefficients for reliability research. J Chiropr Med 2016;15:155-163.

22. Saake M, Breuer L, Goelitz P, et al. Flat detector computed tomography angiography with intravenous contrast application: feasibility for visualization of cerebral arterial vasculature. $J$ Neuroimaging 2013;23:414-420.

23. Struffert T, Deuerling-Zheng Y, Kloska S, et al. Flat detector CT in the evaluation of brain parenchyma, intracranial vasculature, and cerebral blood volume: a pilot study in patients with acute symptoms of cerebral ischemia. Am J Neuroradiol 2010;31:1462-1469.

24. Sabarudin A, Subramaniam C, Sun Z. Cerebral CT angiography and CT perfusion in acute stroke detection: a systematic review of diagnostic value. Quant Imaging Med Surg 2014;4:282-290.

25. Frolich AM, Wolff SL, Psychogios MN, et al. Time-resolved assessment of collateral flow using 4D CT angiography in large-vessel occlusion stroke. Eur Radiol 2014;24:390-396.

26. Menon BK, d'Esterre CD, Qazi EM, et al. Multiphase CT angiography: a new tool for the imaging triage of patients with acute ischemic stroke. Radiology 2015;275:510-520.

27. Garcia-Tornel A, Carvalho V, Boned S, et al. Improving the evaluation of collateral circulation by multiphase computed tomography angiography in acute stroke patients treated with endovascular reperfusion therapies. Interv Neurol 2016;5:209-217.

28. Davis B, Royalty K, Kowarschik M, et al. 4D digital subtraction angiography: implementation and demonstration of feasibility. Am J Neuroradiol 2013;34:1914-1921.

29. Yang P, Niu K, Wu Y, et al. Evaluation of collaterals and clot burden using time-resolved $\mathrm{C}$-arm conebeam $\mathrm{CT}$ angiography in the angiography suite: a feasibility study. Am J Neuroradiol 2017;38:747-752.

30. Struffert T, Lang S, Scholz R, et al. [Radiation dose in cerebral angiography and flat detector CT applications in neuroradiology]. Radiologe 2015;55:654-662. 